\title{
Uso de Software de Gestion Integrada para el logro efectivo de las acitividades empresariales.
}

Use of Integrated Management Software for the effective achievement of business activities.

\section{Cesar Loo Gil}

https://orcid.org/0000-0001-8396-5972

cesarloo@biofab.com.pe

Centro de Investigación BIOFAB

Lima - Perú

\section{Resumen}

Los sistemas integrados de gestión SIG se usan como una nueva herramienta administrativa complementaria a los sistemas de calidad, medioambiente y seguridady salud ocupacional, al unificar algunos elementos comunes. Permitiendo que la interrelación de las empresas con sus entornos se lleve a cabo de manera más amigable y responsable. Los sistemas de planificación de recursos empresariales ERP permiten una mejora integral en el funcionamiento de la empresa. Principalmente se perfecciona el control sobre las operaciones administrativas y financieras, con el fin de mantener un registro actualizado de la facturación, visualizar desde cualquier sitio las facturas pendientes de clientes, monitorear liquidez, saber en línea cuánto se le adeudaa proveedores, controlar el inventario, remuneraciones y ver informes de gestión actualizados. El objetivo general de esta investigación es describir el uso de software de gestión integrada para el logro efectivo de las actividades empresariales. Los resultados que se determinaron fueron el aumento dela eficiencia de la organización, debido a que se disminuyen los problemas operacionales, se logra la optimización de recursos y se aumenta el valor dentro de la cadena productiva. Como conclusión se obtuvo que el uso de software de gestión integrada facilita el logro efectivo de las actividades empresariales.

Palabras Clave: actividades empresariales, sistemas integrados de gestión, software, Planificación de Recursos Empresariales. 


\begin{abstract}
Integrated GIS management systems are used as a new administrative tool complementary to quality, environment and occupational health and safety systems, by unifying some common elements. Allowing the interrelation of companies with their environments to be carried out in a more friendly and responsible way. ERP enterprise resource planning systems enable comprehensive improvement in business operation. Mainly, the control over administrative and financial operations is perfected, in order to maintain an updated record of billing, view pending customer invoices from anywhere, monitor liquidity, know online how much suppliers are owed, control inventory, remunerations and view updated management reports. The general objective of this research is to describe the use of integrated management software for the effective achievement of business activities. The results that were determined were the increased efficiency of the organization, due to the reduction of operational problems, the optimization of resources is achieved and the value within the production chain is increased. As a conclusion, it was obtained that the use of integrated management software facilitates the effective achievement of business activities.
\end{abstract}

Keywords: business activities, integrated management systems, software, Enterprise Resource Planning. 


\section{Introducción}

Las exigencias técnicas que marcan actualmente el ritmo de los mercados nacionales y extranjeros demandan de las empresas soluciones, una de ellas es la gestión integrada. Los tiempos donde las empresas tenían un sistema de gestión financiera, un sistema de gestión de la producción y otros muchos más ya pasaron de moda, lo actual es que se tenga un solo sistema capaz de gestionar todas las necesidades de la empresa y sea manejada como un todo.

Con la implementación de la gestión integrada se concibe a la empresa como un organismo completo e integrado en la sociedad, en el que todo está relacionado, desde el departamento de recursos humanos hasta los procesos de venta, en el que todos tienen que compartir la misma filosofía y los mismos objetivos.Cada uno de los elementos que componen la empresa tienen que ejercer su función teniendo en cuenta a los demás: así es la visión global de la gestión integrada.

Según Pérez y Gardey (2016), las actividades empresariales son muy diversas y dependen de las características de cada organización. Son todas aquellas actividades que lleva adelante una empresa con el objetivo de producir y comercializar los bienes o los servicios que le permiten generar ingresos. Por tanto las tareas que realiza a nivel interno para gestionar su capital y para organizar los recursos materiales y humanos también forman parte de las actividades empresariales.

Las empresas tienen varios criterios para su clasificación una es por el tamaño o número de personas empleadas. Chávez, Campuzano y Betancourt (2018) mencionan que, una micro empresa tiene entre 1 a 9 empleados, una pequeña empresa entre 10 y 49 empleados, la mediana empresa entre 50 y 199 empleados y una gran empresa más de 200 empleados. Otra manera de clasificar a las empresas es por el sector donde se desenvuelven. Así se tiene que pueden pertenecer al sector primario: dedicados a obtener productos de la naturaleza, es el sector agrario, ganadero, pesquero y minero, Sector secundario: dedicados a la transformación de las materias primas, es decir, sector industrial, energético, construcción, entre otros, y el sector terciario: servicios como transporte, comunicaciones, 
comercio, turismo. Sector cuaternario: fomento del conocimiento, servicios de información y creación, investigación y desarrollo e innovación (Peña, 2020).

Las actividades primarias en una empresa, son aquellas que se relacionan con los procesos de fabricación del producto o servicio que se entrega, así como su comercialización, sin terminar en la venta, ya que se brinda un servicio posteriormente, y entre ellas se mencionan las siguientes:

- Logística interna: son las actividades relacionadas con la compra y disposición de insumos o materias primas necesarios para el proceso de producción o elaboración del producto o servicio que ofrece la empresa. Entre las actividades están la recepción de insumos, almacenamiento, distribución de insumos para el proceso de producción, manejo de materiales, control de inventarios de materias primas, manejo de devoluciones de insumos, etc.

- Operaciones: Actividades que participan en el proceso de transformación de insumos en productos o servicios finales, e incluyen las fases de productos intermedios o semiterminados cuando sea necesario. Los aspectos que considera son la planificación y programación de la producción, procesos operativos, mantenimiento del equipo, envasado, control de calidad, manejo de artículos defectuosos.

- Logística externa: Es el traslado, acopio y almacenamiento del producto terminado, así como la organización y planificación de la entrega del producto o servicio hasta el punto de recepción del cliente. Sus actividades son: recepción de productos terminados, almacenamiento y gestión del producto terminado, manejo de inventarios de productos terminados, distribución o entrega en las bodegas del cliente, operación de flota de distribución, pedidos, programación de entregas.

- Marketing y ventas: Están asociadas con la comercialización de los productos o servicios de la empresa. Sus actividades son: selección y manejo de canales de distribución, planificación y ejecución de la publicidad, fuerza de ventas, promociones de venta, establecer políticas comerciales y crediticias, determinar políticas de precios. 
- Servicios posterior a la venta: Son actividades como: garantías, administración de relaciones comerciales con el cliente, políticas de repuestos, vínculos de lealtad entre empresa y cliente, satisfacción del cliente.

Puede ser que una empresa no tenga necesariamente todas estas actividades primarias, se les de otro nombre o incluso que tengan otras distintas a estas, lo importantes es que ayuden a crecer el negocio.

Para Pérez L. (2017) los Sistemas Integrados de Gestión SIG, son un mecanismo que integran las normas de Calidad (ISO 9001), Medio Ambiente (ISO 14001) y Seguridad y Salud Ocupacional (OSHAS 18001), la implementación de este tipo de sistemas permite a la organización coordinar, racionalizar, organizar el trabajo, desarrollando las distintas actividades dentro de la empresa. En la figura 1 se puede apreciar un modelo de SIG.

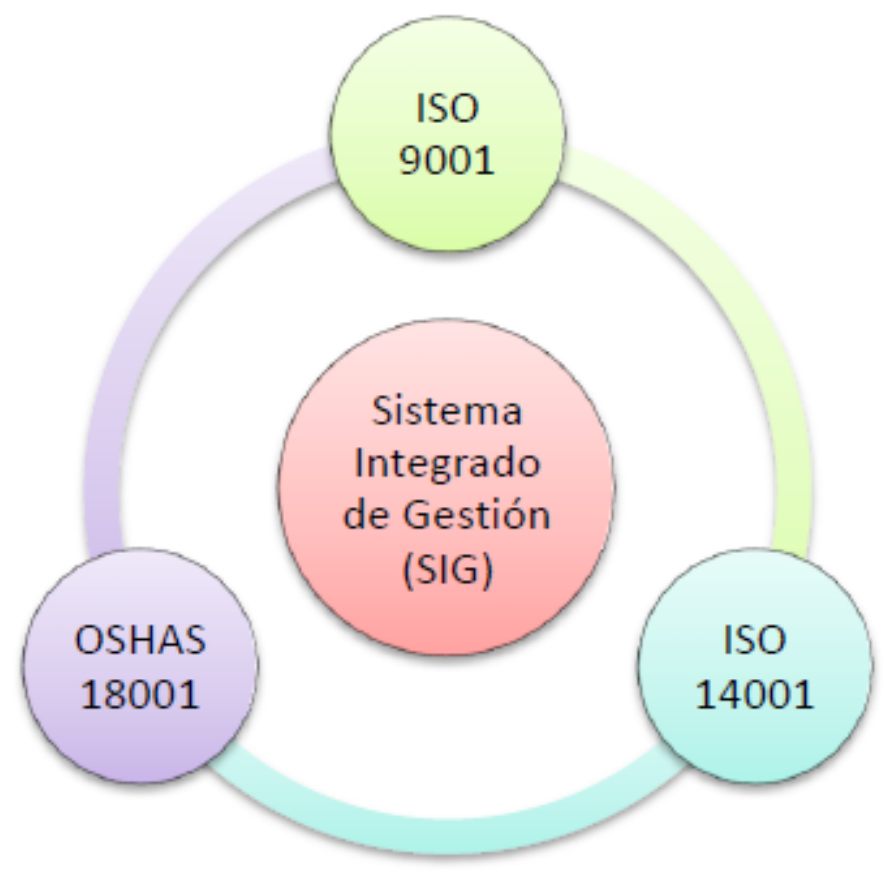

Figura 1. Estructura Sistema Integral de Gestión Fuente: Pérez L. (2017)

Ortiz (2018) menciona que, los sistemas integrados de gestión son uno de los elementos administrativos que ha tenido mayor impulso en los últimos años, debido a la aparición de los estándares internacionales. Pérez, Escoriza y Martínez citado por Ortiz (2018), quienes los consideran como la unión de cada sistema independiente a partir de sus puntos en 
común, con el fin de mantener actualizada la política de la organización. Un punto clave para la integración según Ortiz (2018) es que, define al sistema integrado como el que reúne a los elementos de forma coherente, y que se basa en la satisfacción tanto del cliente como de todas las partes involucradas en la organización.

Debido al auge de los estándares internacionales de calidad, medioambiente y seguridad y salud ocupacional, se ha generado un Sistema Integrado de Gestión enfocado en estas tres normas, denominado HSEQ (Health and Safety, Environment and Quality), representados por los estándares ISO 9001, ISO 14001 y OHSAS 18001.

Los sistemas integrados de gestión se caracterizan por varios aspectos. Conforma un área que se encarga de la gestión del sistema; así mismo existe un responsable de la integración, la documentación es común entre sistemas y las auditorías llevan un plan único. En este sentido Ortiz (2018) menciona que la creación de los sistemas integrados de gestión se debe a una respuesta de las organizaciones a la imposición de requisitos legales por parte de cada gobierno, que son de carácter obligatorio. Otra causa se refiere al antiguo enfoque en la producción en masa a partir de costos de operación elevados y de los efectos indeseados tanto para el entorno de la organización como para las personas involucradas (Osorio y Oviedo, 2013)

Los sistemas integrados de gestión al ser implementados pretenden estandarizar, normalizar, obtener mejoramiento continúo con un único fin y es la excelencia empresarial.

Esta investigación tiene por objetivo general describir el uso de software de gestión integrada para el logro efectivo de las actividades empresariales. La metodología empleada se basó en un diseño bibliográfico de tipo documental.

\section{Metodología}

Esta Investigación se realizó basándose en un diseño bibliográfico de tipo documental. El trabajo se fundamenta en la revisión sistemática, rigurosa y profunda de material documental de cualquier clase, donde se efectúa un proceso de abstracción científica, 
generalizando sobre la base de lo fundamental, partiendo de forma ordenada y con objetivos precisos. (Palella\&Martins, 2010) La investigación documental se concreta exclusivamente en la recopilación de información de diversas fuentes, con el objeto de organizarla describirla e interpretarla de acuerdo con ciertos procedimientos que garanticen confiabilidad y objetividad en la presentación de los resultados (Palella\&Martins, 2010). Para lograr este propósito se utilizaron herramientas como textos, documentos y artículos científicos publicados disponibles en la web.

Los objetivos de esta investigación se basaron en, describir y analizar las actividades empresariales, desarrollar el análisis de los sistemas integrados de gestión en las actividades empresariales, comprender la definición e importancia del software en general y de los sistemas de gestión integrada, analizar el resultado del uso del software de gestión integrada en las actividades empresariales, describir y analizar las características del software ERP en las actividades empresariales.

\section{Resultados y discusión}

Para Raffino (2021) el software es la parte intangible que conforma los programas informaticos y aplicaciones diseñados para cumplir diversas funciones en un sistema. Según su funcion y se clasifican en:

- Software de sistema: programas que dan al usuario la capacidad de relacionarse con el sistema, para ejercer control sobre el hardware. El software de sistema también se ofrece como soporte para otros programas. Por ejemplo: sistemas operativos o servidores.

- Software de programación: programas diseñados como herramientas que le permiten a un programador desarrollar programas informáticos. Se valen de técnicas y un lenguaje de programación específico. Por ejemplo: compiladores o editores multimedia. 
- Software de aplicación: programas diseñados para realizar una o más tareas específicas a la vez, pueden ser automáticos o asistidos. Por ejemplo: videojuegos o reproductores multimedia.

Según Pérez (2017) los componentes del software de un sistema son:

- Procesos: Conjunto de mecanismos de comportamiento o secuencia de pasos que se diseña de manera lógica para lograr resultados específicos con el fin de mejorar la productividad.

- Datos: Los datos son un conjunto de bytes almacenados en archivos, los cuales describen características y en conjunto representan información sobre la que operan los algoritmos, estos datos se codifican y se almacenan de manera sistemática en las bases de datos para ser utilizadas en el futuro.

- Base de datos: Las bases de datos son un conjunto o serie de datos organizados estructuralmente y relacionados entre sí, los cuales son recolectados por los sistemas de información para ser distribuidos entre uno o más equipos, esta información es gestionada mediante software denominados motores de base de datos lo cual permiten a las empresas u organizaciones acceder a la información, actualizarla, modificarla y en algunos motores manteniendo su integridad. (Rodrigo, 2017).

- Sistema de Gestión de Base de Datos: Es un tipo de software muy específico, el cual está dedicado a servir de interfaz entre la base de datos, el usuario y las aplicaciones que utilizan. Está compuesto por tres lenguajes: definición de datos, manipulación de datos y consulta. Esto con el objetivo de garantizar la integridad, disponibilidad y confidencialidad de la información almacenada dentro del sistema.

- Servidores WEB: Los servidores web se encargan de servir o entregar contenido mediante el protocolo HTTP, existen dos tipos de contenido estático y dinámico; El contenido estático es aquel que permanece invariante en el sistema como son archivos de configuración, librerías, imágenes, archivos html entre otros. El contenido dinámico es aquel que se genera dependiendo de las peticiones del cliente y puede variar su contenido dependiendo de múltiples factores como pueden ser la hora en la que se solicita información, el tipo de usuario que la solicita. 
- Sistema de Información: Un sistema de información es un conjunto de varios software (Motores de base de datos, tecnologías, procesos, servidores, aplicaciones personalizadas) que cumplen con uno o varias funciones de acuerdo a los requerimientos con los que el sistema fue diseñado (Tundidor et al. 2018).

En la tabla número 1 se presentan los requerimientos estándar del software del sistema integrado de gestión.

\section{Tabla 1}

Descripción del sistema integrado de gestión SISTEMA DOCUMENTO

\section{DESCRIPCIÓN}

Norma ISO 9001:2015 Requisitos Implementar un sistema de gestión de mínimos de un sistema de gestión calidad, ayuda a gestionar y controlar de calidad.

Gestión de cada uno de los procesos de la organización, cumpliendo con las expectativas y necesidades de sus clientes.

\begin{tabular}{|c|c|c|}
\hline $\begin{array}{c}\text { Gestión } \\
\text { Ambiental }\end{array}$ & $\begin{array}{l}\text { Norma ISO 14001:2015 } \\
\text { Requisitos mínimos de un sistema } \\
\text { de gestión ambiental. }\end{array}$ & 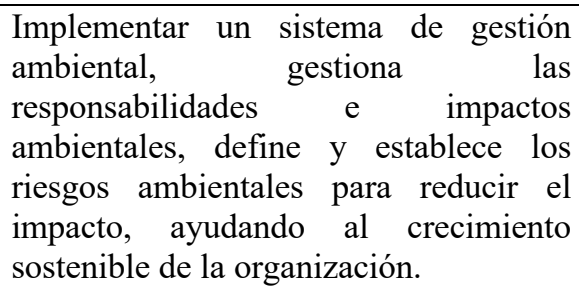 \\
\hline $\begin{array}{l}\text { Gestión de } \\
\text { Seguridady } \\
\quad \text { Salud } \\
\text { Ocupacional }\end{array}$ & $\begin{array}{l}\text { Norma OSHAS 18001:2015 } \\
\text { Requisitos mínimos de un sistema } \\
\text { de gestión de seguridad y salud } \\
\text { ocupacional. }\end{array}$ & $\begin{array}{l}\text { Implementar un sistema de gestión } \\
\text { SSO, brinda a la organización una } \\
\text { estructura correcta y eficaz para } \\
\text { gestionar los riesgos y la salud de sus } \\
\text { trabajadores. }\end{array}$ \\
\hline
\end{tabular}

Fuente: (Pérez ,2017).

Algunas alternativas de software utilizados para los sistemas integrados de gestión son: La Plataforma ISOTools, la Plataforma Tecnológica permite la integración de sistemas normalizados de gestión y modelos de excelencia. ISOTools es una herramienta diseñada para hacer más efectivo el manejo de la documentación, mejorar la comunicación y disminuir tiempos y costos.

Las siglas ERP corresponden al acrónimo de "Enterprise Resources Planning" o "Planificación de recursos empresariales". Son conocidos como sistemas de gestión empresarial o sistemas integrados de gestión, comúnmente se hace referencia a ellos como: ERP. 
Para Zuñiga (2018), un software ERP tradicional o ERP I debe dar cobertura a todas las áreas de negocio internas de una empresa. Algunos de los módulos de negocio que un ERP tradicional debe dar solución son: contabilidad y finanzas, clientes y proveedores, gestión de inventarios, planificación de la producción, gestión de costes, gestión de proyectos, entre otras. La evolución natural de los ERP tradicionales los ERP II tienen su enfoque en la interacción de empresa con su entorno, abarcando participantes fuera de la compañía.

El total acceso a la información que se ha obtenido gracias a internet desencadeno una transformación tecnológica al incrementar el intercambio de información, pero también ha generado cambio en los modelos de negocios de las empresas al generar nuevas relaciones entre proveedores, colaboradores, clientes y empleados, Zuñiga (2018).

Según Heredia (2015), algunas Características de un sistema ERP son:

- Integridad, así como los distintos departamentos dentro de una empresa están relacionados entre sí, los procesos que componen un ERP se encuentran también interrelacionados de forma que la información fluye entre los distintos procesos, evitando la duplicidad de información en áreas estancadas

- Diseño Modular, un ERP está diseñado en bloques funcionales (ventas, compras, producción, finanzas, etc.) que conservando su capacidad de integración, pueden implantarsede manera individual o en bloques. Este hecho permite estrategias de implantación graduales minimizando el riesgo. También permite presupuestos de implantación más dilatados en el tiempo.

- Adaptabilidad, es decir, la capacidad de adaptación del producto a la empresa. Normalmente los ERP están diseñados para que los principales procesos de gestión se puedan parametrizar o configurar según las directrices de negocio marcadas por la empresa (Heredia, 2015)

\section{Tipos y clasificación de sistemas ERP}

Los ERP se pueden dividir según su grado de personalización, en sistemas genéricos, preparametrizados y los individualizados o elaborados a medida de la organización. 
- Según su instalación, Existen tres tipos de formas de alojar un sistema ERP; localmente, en la nube o una combinación de ambos. Local: Servidores propios dentro de la organización, en los cuales la información fluye solamente dentro de la red que soporta el servidor. Nube: La información se almacena en el servidor del proveedor del servicio y no es necesario tener servidores dentro de la empresa. (Logroño, 2017)

- Según su código abierto: Esta solución resulta interesante para empresas que cuenten con un equipo informático amplio y experimentado, puesto que permite desarrollar e integrar aplicaciones y módulos propios al software de gestión. Código privado: No permiten el acceso a su código fuente y solo puede ser modificado por sus desarrolladores. (Oltra, 2017)

Ortiz (2018), menciona que, el mayor impacto de los sistemas integrados de gestión reportados es la eficiencia de la organización, debido a que se disminuyen los problemas operacionales, se articula la estrategia con el desempeño de los procesos, se logra la optimización de recursos y se aumenta el valor dentro de la cadena productiva.

Adicionalmente Flórez (2015) identifica otros impactos relacionados con el personal, con la mejora en las condiciones de trabajo, eliminación de barreras entre departamentos, definición de responsabilidades y el entendimiento del sistema integrado. También se encuentra el apoyo del sistema integrado en el mejoramiento continuo, al contribuir en los métodos de trabajo, uso de resultados de auditorías, aumento de indicadores y la disminución de no conformidades (González, 2019)

La implementaciónde los sistemas integrados de gestiónpueden ser percibidos de formas distintas, dependiendola actividad que realiza cada organización. En las industrias evaluadas por Oviedo y Osorio (2015) se encontró que el sector de hidrocarburos prefiereintegrar sistemas por cuestiones de imagen y reducciónde accidentalidad, mientras que el sector alimenticiopor requisitos legales y de procesos.

La integración de sistemas pasó de seruna cuestión documental, o de requisitos similares, a una estructura más compleja donde los procesos, lasfunciones, responsabilidades y 
compromisos de ladirección son fundamentales para el éxito del sistema (Rebelo, Santos, y Silva, 2016).

El crecimiento mundial de empresas que deciden implementar un sistema de información como los ERP, se ven beneficiadas gracias a la automatización y simplificación de los procesos, integración de áreas funcionales, y poseer una base de datos centralizada que permita obtener datos fidedignos y únicos (Zuñiga, 2018). Por otra parte, es muy importante señalar que los cambios que se deben realizar dentro de la organización son fundamentales para un correcto desarrollo de las funciones del software.

Según González (2019) muy pocas organizaciones destinan parte de esos recursos a la gestión empresarial. Esto se debe al desconocimiento, ya que la información es uno de los activos más valiosos y su buen manejo es transcendental, pues les entrega sentido a los datos percibidos para finalmente ser usado como conocimiento en el proceso de toma de decisiones.

La utilización de tecnologías basadas en software privado, genérico y en nube brinda facilidades en la implementación, creando un ambiente seguro, robusto y con costos examinados previamente, (Zuñiga, 2018).

Las principales características que debe presentar un software ERP son la integralidad para que la información fluya entre los distintos departamentos, un diseño modular que permita ir incluyendo estrategias de implantación graduales minimizando el riesgo. También permite presupuestos de implantación más dilatados en el tiempo. (Heredia, 2015).

\section{Conclusiones}

Las actividades empresariales son todas aquellas le permiten a la empresa producir y comercializar bienes o servicios que le permiten generar ingresos, pueden ser de origen interno o externo, una empresa puede ser clasificada de varias maneras una es por la cantidad de personal empleado, pudiendo ser micro, pequeña, mediana o gran empresa, otra 
manera es por el sector donde se desempeña, pudiendo ser del sector primario, secundario, terciario o cuaternario.

Los sistemas integrados de gestión SIG le permiten a las empresas operar de una manera más integral al tratar de balancear las actividades internas con las externas, tomando en cuenta la relación con el cliente, al servirle a través de la atención postventa. Procurando formalizar una relación de lealtad.

El Software toma una relevancia muy importante pues permite la creación de programas, sistemas, aplicaciones, comunicaciones, y manejo de data, de una manera segura, rápida, confiable y económica, permitiendo que se desarrollen relaciones más transparentes entre los distintos departamentos que conforman la empresa, incluso pudiendo agregar otras formas de llegar a los clientes, a través del e-commerce.

El uso de software de gestión integrada permite a los gerentes de las empresas tomar decisiones más acertadas debido a la versatilidad en el uso de los datos recolectados. Los sistemas integrados de gestión se usan como unanueva herramienta administrativa complementaria alos sistemas de calidad, medioambiente y seguridady salud ocupacional, al unificar algunos elementoscomunes.

Las funciones o capacidades del software ERP se extienden no solo a áreas como producción, ventas, finanzas o distribución, sino que engloban aspectos característicos de cada sector industrial o industria en particular. Permitiendo tener soluciones de acuerdo al tamaño y cantidad de usuarios de la compañía en el área Finanzas, área Comercial, área Operaciones y en el área de Gestión de Recursos Humanos.

\section{Referencias Bibliográficas}


Chávez, G., Campuzano, J., \& Betancourt, V. (2018). Las micro, pequeñas y medianas empresas. Clasificación para su estudio en la carrera de Ingeniería en Contabilidad y Auditoría de la Universidad Técnica de Machala. Conrado , 14 (1), 247-255.

Flórez, A. (2015). Importancia de implementar en las organizaciones floricultoras de Colombia un sistema integrado de gestión como beneficio para incursionar en el comercio internacional. Bogotá, Colombia: Trabajo especial de grado de la Universidad Militar Nueva Granada para optar al título de Administradora en Seguridad y Salud Ocupacional.

González, W. P. (2019). Incidencia de la cultura organizacional en la eficacia de los sistema integrados de gestión. Bogotá, Colombia: Artículo de investigación de la Universidad Militar Nueva Granada .

Heredia, F. (2015). Implantación de un Sistema SAP ERP en el sector farmacéutico. Barcelona: Universitat politécnica de Catalunya.

Logroño, E. (2017). Análisis de los servicios Cloud Computing para una gestión empresarial eficaz. PONTIFICIA UNIVERSIDAD CATÓLICA DEL ECUADOR, FACULTAD DE INGENIERÍA. Quito: Trabajo especial de grado de la Pontificia Universidad Católica del Ecuador para optar al título de Maestría en redes de comunicación.

Oltra, R. (2017). FSw ERP (Free Software ERP). Diferencias con el ERP propietario. Valencia.

Ortiz, Y. (2018). EL IMPACTO DE LOS SISTEMAS INTEGRADOS DE GESTIÓN HSEQ EN LAS ORGANIZACIONES DE AMÉRICA LATINA: UNA REVISIÓN SISTEMÁTICA. Revista Chilena De Economía Y Sociedad , 12 (2), 77-93.

Osorio, K., \& Oviedo, J. (2013). Sistemas integrados de gestión en las empresas de Colombia. Revista Gremios, 7. 
Palella, S., \& Martins, P. (2010). Metodología de la Investigación Cuantitativa. (2da ed.). Caracas, Venezuela: FEDEUPEL.

Peña, 1. (8 de 09 de 2020). Clasificación de las empresas: Tipos y Definición. Recuperado el 28 de Junio de 2021, de https://www.billin.net/blog/clasificacion-empresas/

Pérez, J., \& Gardey, A. (2016). Definición de Actividad Empresarial. Recuperado el 28 de Junio de 2021, de https://definicion.de/actividad-empresarial/)

Pérez, L. (2017). Implementación de un sistema de gestión empresarial para TSMTSAS. Bogotá, Colombia: Monografía para optar al Título de Ingeniera en Automatización de la Universidad de la Salle.

Raffino, M. E. (21 de enero de 2021). Concepto de Software. Recuperado el 29 de Junio de 2021, de https://concepto.de/software/\#ixzz6zBU1TXB2

Rebelo, M. F., Santos, G., \& Silva, R. (2016). Integration of management systems: Towards a sustained success and development of organizations. Journal of Cleaner Production 127 (127), 96-111.

Rodrigo, S. (2017). Introducción de las tecnologías de la información y de la comunicación a la gestión empresarial. Trabajo especial de grado de la Universidad de Jaén.

Tundidor, L., Nogueira, D., Médina, A., \& Serrate, A. (2018). Requerimientos de los sistemas informativos para potenciar el control de gestión empresarial. Ciencias Holguín, 24 (1), 43-56.

Zuñiga, G. (2018). EVALUACIÓN Y SELECCIÓN DE SISTEMA DE PLANIFICACIÓN DE RECURSOS EMPRESARIALES "ERP" PARA ZOOMGRAFIC. Universidad Tecnica Federico Santa Maria, Departamento de Ingenieria Comercial. Valparaiso: Trabajo especial de grado de la Universidad Técnica Federico Santa María para optar al Título de Ingeniero Comercial . 